\title{
Survey of Text Messaging System using Bluetooth and Wi-Fi
}

\author{
Zainab Pirani,PhD \\ Dept. Computer \\ Engineering \\ M.H.S.S.C.O.E
}

\author{
Bilal Zaveri \\ Dept. Computer \\ Engineering \\ M.H.S.S.C.O.E
}

\author{
Rafiaan Shaikh \\ Dept. Computer \\ Engineering \\ M.H.S.S.C.O.E
}

\author{
Ejaj Shaikh \\ Dept. Computer \\ Engineering \\ M.H.S.S.C.O.E
}

\begin{abstract}
Communication using text-based messaging applications have seen an enormous rise in popularity over the last few years. With growing popularity, dependency on them has increased. It is used for staying in touch with friends, family and sometimes for doing business. But text-based messaging largely depends on internet. If internet is not available due to some reason then communication is not possible. There is a need to find an alternate method to overcome this problem. In this paper we are exploring various method that can be used for text- based communication other than using internet. We are also reviewing various problems in those methods such as congestion and inefficiency of the network. Further we are also reviewing different techniques to overcome this problem.
\end{abstract}

\section{Keywords}

Messages, Applications, Internet, Communication, congestion, inefficiency, network.

\section{INTRODUCTION}

The use of smartphones has been increasing rapidly from the world's population is projected to use a smartphone by 2018, up from about 10 percent in 2011 [1]. Smart phone has introduced new way of communicating with people. Now messaging application has become a necessity. People use it to stay connected with friends, family or sometimes for doing business. People can do one to one or group communication using it. But messaging application relies on internet to work. If a person has active internet connection then he/she can stay in touch with people. If internet is not available and a person wants to send messages then he/she won't be able to communicate. To overcome this problem various methods were proposed and experimented such as use of built in Bluetooth and Wi-Fi of devices for short distance communication. These methods are not much in use and are still under research. Communication can be possible using Bluetooth by connecting two nearby devices. Using Wi-Fi, a mesh network can be created so that more than two devices can stay connected with each other [5]. But Wi-Fi mesh networks also have many problems. In this paper, a survey has been done on different works by different researchers to overcome the problems of wireless mesh network. Many researchers are working to increase the efficiency and to optimize the network. Efficiency of the mesh network can be increased by adopting an efficient routing algorithm for finding the best path to send the message to the receiver. Another way to communicate is by using a hybrid model where both Wi-Fi and Bluetooth can be used. There is also a way of communication for messaging application here wireless mesh network can be combined with cellular internet to form a heterogeneous network where both the drawbacks of internet and wireless mesh networks can be overcome to some extent and efficiency can be increased[7].

Security is also a major issue in communication via Bluetooth and Wi-Fi. Many researches are done to overcome some of the issues. Messages send via Bluetooth and Wi-Fi can be encrypted first before sending to ensure that only the recipient can read it. Also, in group communication a protocol can be developed to prevent a new member in the network from reading the previous messages [8].

\section{RELATED WORK 2.1 Paper Based Survey}

\subsubsection{Text Based Communication Using Bluetooth}

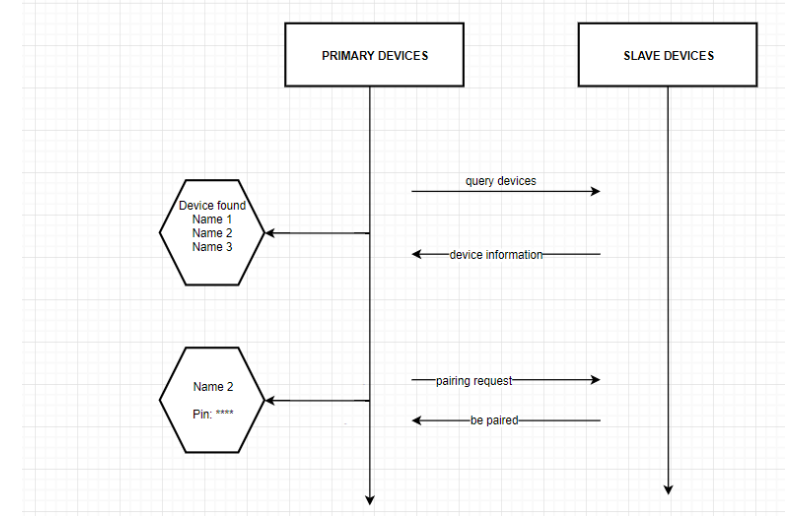

Fig.1 Architecture of text messaging system using Bluetooth

In [2], the author implements the text-based messenger using Bluetooth of the devices as shown in the figure 1. Bluetooth based messaging system sends message from one device to other by using built in Bluetooth technology in device.

For communication using Bluetooth based messaging, it first checks whether the Bluetooth of the device in ON or OFF. If it is off then the system will enable the Bluetooth of the device. Once it is on it checks all the available devices in its range. It then displays all the available devices to the user. Once the user selects the desired device which they want to communicate, the system will connect to that particular device and initiates the communication.

\section{Merits}

1- Easy to connect

2- No cost

3- Easily available

\section{Demerits}

1- Range is limited as compared to Wi-Fi.

2- Only one to one connection can be established, no groups can be formed. 


\subsubsection{Text Based Communication Using Wi-Fi}

In [5], author implements the text-based messenger using WiFi direct of devices. Wi-Fi direct is used to create ad hoc network between devices. When the devices are connected a simple client / server construction is used to send messages over the network. Each smartphone has a server where other smartphones can connect to. This way each smartphone acts as a node where other devices can connect to. Also, every smartphone has a collection of sockets to other devices. Every time this smartphone sends a message it will be sent to all the devices it is connected to. Also, every message received will be sent over the same sockets. This way a message from another device is transmitted over the network. One of the ways the messages can be send through the network is flooding: every device broadcasts the messages received. One of the problems can be that a device that sends a message will receive the message back from the devices connected. This way a loop is created: one message will be broadcast over and over. One of the ways to fix this is to give an id to every message. This way a device must look up the id in a list from id's send. This will prevent that the network keeps on broadcasting one message. Some way to prevent the constant looping of the messages over the network must be used in the application.

\section{Merits}

1- Can form network group.

2- Range is more as compared to Bluetooth.

\section{Demerits}

1- Consumption of power is more as compared to Bluetooth.

\subsubsection{Text Based Communication Using Hybrid System}

Hybrid network is defined as a system consisting of two or more technologies. In this paper we came across a different ideas and system that uses two technologies to communicate [7]. The paper [7] proposes the idea of sharing file by using Wi-Fi and Bluetooth in an efficient manner without any loss of data and reconnecting if the connection is lost. Though this paper proposes the idea of sharing files it can also be used as a text messenger.

When devices are connected to each other seamless service manager (SSM) sender communicates with file manager of receiver. When file to transmit the (SFS) chooses the channel for transmission i.e. Wi-Fi or Bluetooth according to the network connected. When sender sends through Bluetooth the device first searches for the devices which are connectable and connects through the MAC address of the receiver. When forward through Wi-Fi it uses UDP for sending.

\subsubsection{Text Based Communication Using} Heterogeneous System.

In [12], author proposes an idea of heterogeneous network architecture in which cellular internet and Wi-Fi mesh network are combined to overcome drawbacks of each of them as shown in figure 2. By introducing a new routing protocols, they are evaluating the cost of transmitting traffics through each network. The simulation results show that the proposed network achieves up to $200 \%$ more throughput when compared to internet only and Wi-Fi only networks. The architecture of the network manages the different wireless devices as a part of single virtual network. The cellular internet is utilized to avoid congestion in Wi-Fi nodes and high interference path in Wi-Fi mesh network and Wi-Fi mesh network offloads the load of the internet and reduces the cost of using it. Further this type of network can be used in future to connect the Internet of Things networks and employed to provide infrastructure for smart homes and smart cities.

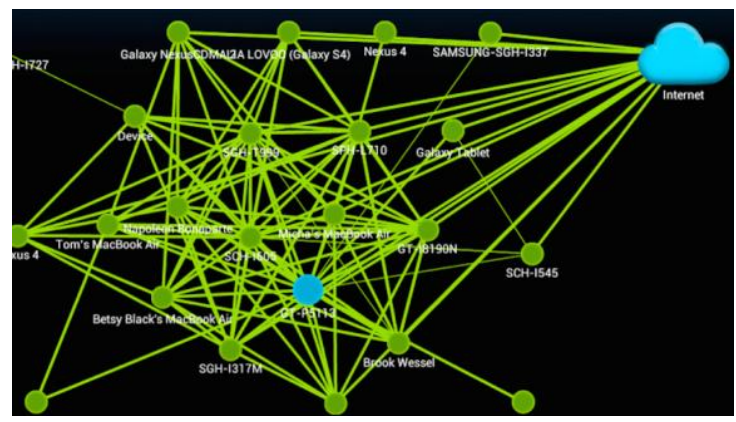

Fig.2 Connection in heterogeneous messaging system

\subsubsection{Summary}

Table one shows the advantage and disadvantage of the Wireless Technologies.

Table 1: Advantages and Disadvantages Wireless Technologies

\begin{tabular}{|l|l|l|}
\hline & Wi-Fi & Bluetooth \\
\hline Range & $100 \mathrm{~m}$ & $10 \mathrm{~m}$ \\
\hline Throughput & $54 \mathrm{Mb} / \mathrm{s}$ & $1 \mathrm{Mb} / \mathrm{s}$ \\
\hline $\begin{array}{l}\text { Power } \\
\text { Consumption }\end{array}$ & $400 \mathrm{~mA}+$ & $40 \mathrm{~mA}$ \\
\hline $\begin{array}{l}\text { Number of } \\
\text { Nodes }\end{array}$ & Unlimited & 8 \\
\hline
\end{tabular}

\subsection{Application Survey}

Fire Chat is a messaging application which can work without an Internet connection or cellular network coverage. It uses Bluetooth and Wi-Fi technology of devices for communication. It creates a wireless mesh network as shown in figure 3 over a short distance between devices that are using it. It can also work on internet to communicate with devices. When the application is launched it requests the user to switch on Bluetooth and Wi-Fi of the device. Firechat automatically connects the devices directly with one another when two devices are less than $200 \mathrm{ft}$. apart. User can send both public and private messages through this new kind of network. Public messages can be seen by everyone in the network, while private message can only be seen by the sender and recipient of the message. This message is encrypted in network so no one else can read them.

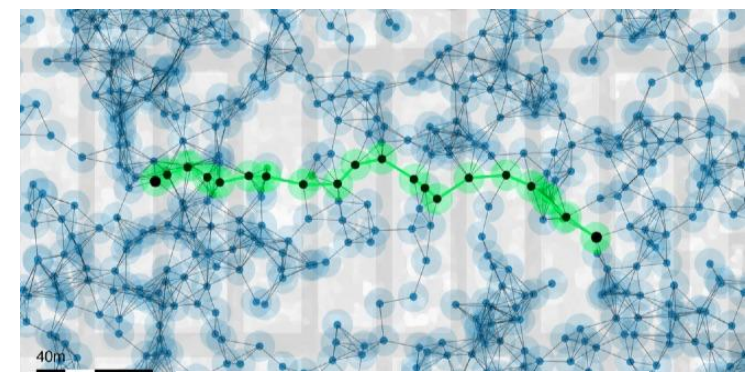

Fig.3 Linking of devices in Firechat

\subsection{Technical Survey}

Author in paper [8] has proposed a way of secure group communication in wireless Mesh network (WMNs). They focused on providing data confidentiality for group communication WMNs. They created a new protocol 
framework called secure group overlay multicast (SeGrOM). This protocol hides the previous communication from newly joined members and protecting future information from the member who are no longer part of the group, thus protecting sensitive information and providing confidentiality to the members without affecting performance of the group.

In paper [12] author proposes an idea of android application for sharing the file without any loss if data by using Wi-Fi and Bluetooth. They proposed the idea that SFS will transmit and receives the file simultaneously. The paper primarily focuses on sharing the files which can be used to modify by using their idea as text-based communication application.

The author in paper [9] focuses on the congestion problem in wireless mesh networks. Congestion is a serious problem specially when used in the automation context with its fixed data rate application. The author tries to solve by using reservation mechanism. They present a routing Architecture for wireless mesh network which prevents congestion using bandwidth reservation. The architecture also includes a calibration process to achieve a high utilization of the available bandwidth. They also presented an approach on how the results of the initial calibration can be continuously updated via passive measurements and network stimulation.

The author in paper [10] focuses on variety of routing protocols that are used in wireless mesh network and also analyse performance of each of them. The parameters used to analyse performance are load balancing, packet delivery ratio, congestion, network overhead, throughput and mobility of nodes. They presented detailed study of proactive routing protocols like DSDV, CGSR and OLSR, reactive routing protocols like DSR, AODV, LQSR and TORA protocols and hybrid protocols such as ZRP. Different protocols are available and can be used according to the requirement of the network. They provided a guideline showing which routing protocols will be efficient, depending on the network and other conditions.

\section{PROPOSED SOLUTION}

It was observed that a solution can be created to overcome some of the drawbacks of the existing system that are surveyed in this paper. The solution is based on hybrid network where it will be using Bluetooth and Wi-Fi along with cellular internet. The system will be able to communicate in short distance using only Bluetooth and Wi-Fi and also will be able to communicate via cellular internet.

\section{CONCLUSION}

In this survey paper we try to briefly describe various type of technique that can be used for text-based messaging such as using internet, $\mathrm{Wi}-\mathrm{Fi}$, Bluetooth or by creating hybrid network of Bluetooth and Wi-Fi. There is an advantage of Wi-Fi and Bluetooth as they are in built technology in devices and they don't cost anything to use them. It was also found that Wi-Fi and Bluetooth can be used along with internet to increase efficiency of the whole network. But using $\mathrm{Wi}-\mathrm{Fi}$ and Bluetooth for communication creates custom network so there can be various problems like congestion, inefficiency and data loss. So, to overcome this issue we came across many paper which were focused on solving this problem.

In future, technology of $\mathrm{Wi}-\mathrm{Fi}$ and Bluetooth can be used more efficiently as new versions of wifi and bluetooth such as Bluetooth 5.0, Wifi aware, etc. will become more common and will increase the range and efficiency of the network. Various technique can be developed to decrease time for connecting devices. Using machine learning, efficiency of the network can be further increase and various problems of the network can be solved.

\section{ACKNOWLEDGEMENT}

A paper is never the outcome of a single person's effort. It is said that "The team work will make the dream work". This paper is a result of various thoughtful minds that are integrated to produce a resourceful survey paper which intend to benefits of people. It is natural that we feel indebted to several people for having made this paper possible. This paper was prepared under the guidance of Dr. Zainab Pirani.

\section{REFERENCES}

[1] https://www.statista.com/statistics/330695/number-ofsmartphone-users-worldwide.

[2] Nikita Mahajan, Garima Verma, Gayatri Erale, Sneha Bonde, Divya Arya "Design of Chatting Application Based on Android Bluetooth". IJCSMC, Vol. 3, Issue. 3, March 2014, pg.712 - 717.

[3] Aishwarya S Bote, Nitish Ghare, Pravin Kumar Rahurkar, Mandar Latkar, "Bluetooth Chat Application: Bluez". Marathwada Mitra Mandal's College Of Engineering, Karve Nagar, Pune, Maharashtra, India.

[4] Arnab Banerjee, "A Bluetooth Messenger Application". Department of Computer Science National Institute of Technology, Rourkela May 11, 2015.

[5] Matthijs Gielen, "Ad hoc networking using Wi-Fi during natural disasters: overview and improvements". University of Twente.

[6] Mr. Boopesh kumawat, Sudhendra Pal Singh, Chandra Prakash Verma, "Intranet Based Messaging Service on Android Smartphones and Tablets". Volume 3, Issue 7, July 2013 ISSN: 2277 128X International Journal of Advanced Research in Computer Science and Software Engineering.

[7] Ajita Arvind Mahapadi; Prof.Soumitra Das, "Messaging System for Hand Held Devices Using Wi-Fi/Bluetooth Network". Multidisciplinary Journal of Research in Engineering and Technology, Volume 2, Issue 1, Pg.365369.

[8] Jing Dong, Kurt Ackermann, Cristina Nita-Rotaru, "Secure Group Communication in Wireless Mesh Networks". Department of Computer Science, Purdue University 305 N. University St., West Lafayette, IN 47907 USA.

[9] André Herms, Edgar Nett, Stefan Schemmer, "Real-Time Mesh Networks for Industrial Applications". The International Federation of Automatic Control Seoul, Korea, July 6-11, 2008.

[10] Muhammad Mehran Ajmal, Khalid Mahmood, Sajjad A Madani, "Efficient Routing in Wireless mesh network by Enhanced AODV".

[11] K.P. Vijayakumar, P. Ganeshkumar and M. Anandaraj, "Routing Algorithm in Wireless Mesh Network". Department of IT, PSNA College of Engg. \& Tech., Dindigul, TamilNadu, India.

[12] Ahmed Al-Saadi, Rossitza Setchi, Yulia Hicks, Stuart Allen, "Routing Protocol for Heterogeneous Wireless Mesh Networks". 10.1109/TVT.2016.2518931, IEEE Transactions on Vehicular Technology. 
[1] Pedro Lopez, Diego Garca, Sergio Almagro, Juan J.Alcaraz, Fernando Cerdan , "Development of a cooperative application for sending SMS on WiFi mobile phones". The Second International Conference on Mobile Ubiquitous Computing, Systems, Services and Technologies.

[2] Elmurod Talipov, Yohan Chon, and Hojung Cha, Member, IEEE, "Content Sharing over SmartphoneBased Delay-Tolerant Networks". International Journal of Research Studies in Science, Engineering and Technology.
[3] Yi Li, Lili Qiu, Yin Zhang, Ratul Mahajan, Zifei Zhong, Gaurav Deshpande, Eric Rozner, "Effects of Interference on Wireless Mesh Networks: Pathologies and a Preliminary Solution".

[4] Pedro López, Diego García, Sergio Almagro, Juan J. Alcaraz, Fernando Cerdan, "Development of a cooperative application for sending SMS on WiFi mobile phones". The Second International Conference on Mobile Ubiquitous Computing, Systems, Services and Technologies. 